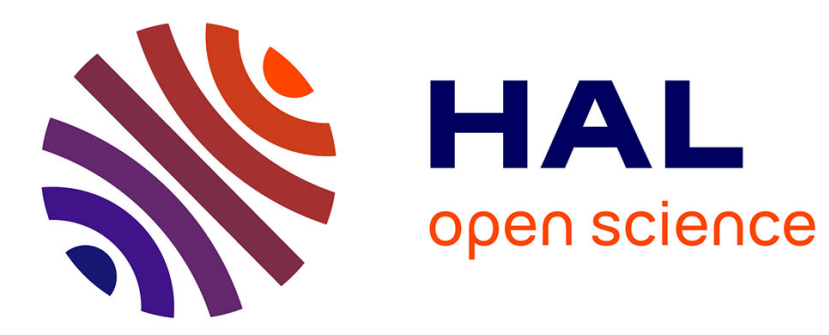

\title{
A preliminary design formula for the strength of stiffened curved panels by design of experiment method
}

Khan Le Tran, Cyril Douthe, Karam Sab, Julien Dallot, Laurence Davaine

\section{To cite this version:}

Khan Le Tran, Cyril Douthe, Karam Sab, Julien Dallot, Laurence Davaine. A preliminary design formula for the strength of stiffened curved panels by design of experiment method. Thin-Walled Structures, 2014, 79, pp. 129-137. 10.1016/j.tws.2014.02.012 . hal-00960346

\section{HAL Id: hal-00960346 https://hal.science/hal-00960346}

Submitted on 18 Mar 2014

HAL is a multi-disciplinary open access archive for the deposit and dissemination of scientific research documents, whether they are published or not. The documents may come from teaching and research institutions in France or abroad, or from public or private research centers.
L'archive ouverte pluridisciplinaire HAL, est destinée au dépôt et à la diffusion de documents scientifiques de niveau recherche, publiés ou non, émanant des établissements d'enseignement et de recherche français ou étrangers, des laboratoires publics ou privés. 


\title{
A preliminary design formula for the strength of stiffened curved panels by design of experiment method
}

\author{
K. L. Tran ${ }^{\mathrm{a}}$, C. Douthe ${ }^{\mathrm{b}, *}$, K. Sab ${ }^{\mathrm{c}}$, J. Dallot ${ }^{\mathrm{a}}$, L. Davaine ${ }^{\mathrm{d}}$ \\ ${ }^{a}$ SNCF, Direction de l'Ingénierie, 6, av. F. Mitterrand, F-93574 La Plaine St Denis \\ ${ }^{b}$ Université Paris-Est, IFSTTAR, F-77447 Marne-la-Vallée \\ ${ }^{c}$ Université Paris-Est, Laboratoire Navier (UMR 8205), ENPC, IFSTTAR, CNRS, \\ F-77455 Marne-la-vallée Cedex 2 \\ ${ }^{d}$ Ingérop, Expertises et Structure, 168/172, bd de Verdun, F-92408 Courbevoie
}

\begin{abstract}
In bridge construction, the use of stiffened plates for box-girder or steel beams is common day to day practice. The advantages of the stiffening from the economical and mechanical points of view are unanimously recognized. For curved steel panels, however, applications are more recent and the literature on their mechanical behaviour including the influence of stiffeners is therefore limited. Their design with actual finite element software is possible but significantly time-consuming and this reduces the number of parameters which can be investigated to optimise each panel. The present paper is thus dedicated to the development of a preliminary design formula for the determination of the ultimate strength of stiffened cylindrical steel panels. This approximate formula is developed with help of a design of experiment method which has been adapted from the current statistical knowledge. This method is first presented and its feasibility as well as its efficiency are illustrated through an
\end{abstract}

\footnotetext{
${ }^{*}$ Corresponding author

Tel: +33181668136; E-mail:cyril.douthe@ifsttar.fr
} 
application to the reference case of unstiffened curved panels. Then, the case of stiffened curved panels is investigated and a preliminary design formula is developed. The ease of use of this formula for preliminary design is finally illustrated in a cost optimisation problem.

Keywords: Design of computer experiments, Response surface, Cylindrical curved panels, Stiffeners, Stability, GMNIA.

\section{Introduction}

The interest of stiffening steel plates or panels to increase their strength 3 under compression has been known for almost a century [1]. In the field 4 of structural engineering, the use of such panels is a common practice, for 5 example in bottom flanges of box-girder bridges. Recent developments of 6 the curving process allowed for the use of curved panels in civil engineering 7 structures where they offer attractive aesthetic and aerodynamic possibilities.

8 The verification of these panels is yet difficult due to a lack of specifications, 9 especially in European Standards: EN 1993-1-5 [2] gives specifications for 10 flat or slightly curved panels with the condition $R \geq R_{\text {lim }}=b^{2} / t_{p}$ (where ${ }_{11} R$ is the curvature radius of the panel, $b$ its width and $t_{p}$ its thickness) and

the curved panels in bridges have characteristics exactly between these two conditions, as illustrated in the case of the Confluences bridge in Angers, France 2011 (Fig. 1), whose radius $R=80 \mathrm{~m}$ is much smaller than the limit of EN 1993-1-5: $R_{\text {lim }}=1440 \mathrm{~m}$ (with $b=4.8 \mathrm{~m}$ and $t=16 \mathrm{~mm}$ ) and for which EN 1993-1-6 is not applicable neither because these curved flanges are not full revolution cylinders. 


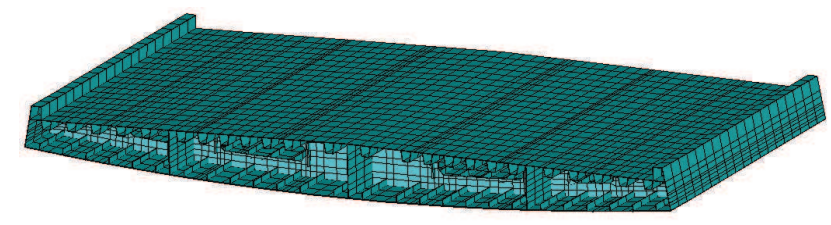

Figure 1: Stiffened curved panel of the Confluences Bridge in Angers (France, 2011)

From an academic point of view, the articles related to the buckling theory of curved panels are not so numerous due to the complexity of the studied problem and also due to its late application in the bridge construction. First investigations were conducted in the forties by Batdorf \& Schildcrout [4] and Schildcrout \& Stein [5] who showed that the stiffeners and the curvature increase the critical buckling strength. A state of the art on curved stiffened panels was then proposed by Becker [6] in 1958 in its handbook on structural stability. Based on experimental results (provided by Gall [7], Lundquist [1] and Ramberge et al. [8]), he confirmed that, when a stiffened flat panel is bent to a circular curve, its buckling stress is slightly increased (around $6 \%$ for the tested specimen which is relatively few compared to the effect of stiffening alone or curvature alone). More recent parametric studies based on numerical examples and the finite element modelling (e.g. Cho et al. [9], Khedmati \& Edalat [10] or Park et al. [11]) investigated and quantified the influence of the main parameters on the ultimate strength of curved stiffened plates. They however did not lead to a practical criterion for the evaluation of the resistance of such panels which is therefore still an open question.

In a former study, the authors [12] had investigated the case of unstiffened cylindrical curved panels under axial compression and established a set 
of formulas for the evaluation of the ultimate strength (which were confirmed by [13]). These semi-analytic formulas had been fitted on a total of $524 \mathrm{com}-$ binations of the main parameters. Each calculus involved Geometrical and Material Non-linearity with Imperfection Analysis (GMNIA) and required between 5 and 10 minutes depending on the refinement of the mesh. Considering the fact that in the case of stiffened panels the number of parameters is considerably larger, re-employing the same methodology seemed unrealistic. It appeared hence that there is a need for a robust strategy for the choice of the set of tested models and for the measure of the approximated model accuracy. Such a strategy exists for the design of physical experiments as well as for that of computer experiments, they are known as "design of experiments methods".

In the following, the authors present thus first the characteristics of computer experiment strategies. Afterwards the feasibility and ease of use of the methodology as well as its efficiency are illustrated through an application to the reference case of unstiffened curved panels. Then, the case of stiffened curved panels is investigated and a preliminary design formula is developed. The interest of this formula for early stages of design is finally illustrated by a short example of cost optimisation.

\section{Design of computer experiments}

\subsection{Background of the design of experiments method}

Design of experiment (DOE) methods exist since the beginning of scientific experiment. The first formal theory for the design of experiments in a "modern sense" was published by Fisher [14] in the 1920s and 1930s, 
while working on improving agricultural yield. Since the $1940 \mathrm{~s}$, various researchers have promoted and developed the use of experiments strategies in many other areas [15]. In the late 1970s, the theory of Taguchi [16] on quality improvement made the design of experiment widely used in the industrial environment. In the past 20 years, advances in computational power have led to the study of physical process through computer simulated experiments, which tends to replace physical experiments in cases where the number of variables is too large to consider performing a physical experiment or where it is simply economically prohibitive to run an experiment on the scale required to gather sufficient information.

Computer experiments differ from traditional physical experiment in their deterministic character, meaning that the computer produces identical answers for the same set of experimental parameters. The error in computer experiments is no longer due to random effects which derive from the variability in experimental units, the order of experiments or the locations of the tests. However, it was shown that in many cases, the systematic error between a deterministic model and its approximation has a normal distribution, so that standard statistical techniques can still be applied [17]. Several authors $[17,18,19]$ also insisted on the fact that the selection of parameter's values for computer runs is still an experimental design problem of primary importance, especially considering the quantification of uncertainty of the model on a statistical point of view. Indeed, as not every combination of parameters can be tested, uncertainty and hazard enter the deterministic process through the choice of tested combinations. The design of a computer experiment is hence at the border of a physical and a statistical problem 
which specificities are emphasized in the following section.

\subsection{General progress of the design of computer experiment method}

Schematically, a numerical model can be considered as a process: the user specifies the combinations of (input) variables to the computer simulator from which the responses (output) are generated. Fig. 2 illustrates this process in the simple case where there are only two input values $\left(X_{1}\right.$ and $\left.X_{2}\right)$ and one response $Y$. Each variable can take a value from "low" to "high". The set of all domains of variation forms the "region of interest". In correspondence with each input variable $\left(X_{1}^{i}, X_{2}^{i}\right)$, the computer program will provide one result $Y^{i}$. A set of $n$ responses will then generate by extrapolation a response surface. In practice, the explicit formula for this surface is not known. The aim of DOE method is to provide approximated models (response surfaces) that are sufficiently accurate to replace the true response and can be used to facilitate design space exploration, optimisation or reliability analyses.

The general steps of computer experiments are generally similar to those encountered in classical experiments [20] and can be summarized as follow:

- Step 1: Statement of the problem.

- Step 2: Choice of the model for the response surface.

- Step 3: Selection of the input data points.

- Step 4: Evaluation of the approximated model.

- Step 5: Validation of the accuracy of the response model.

- Step 6: Selection of most significant terms and conclusion. 


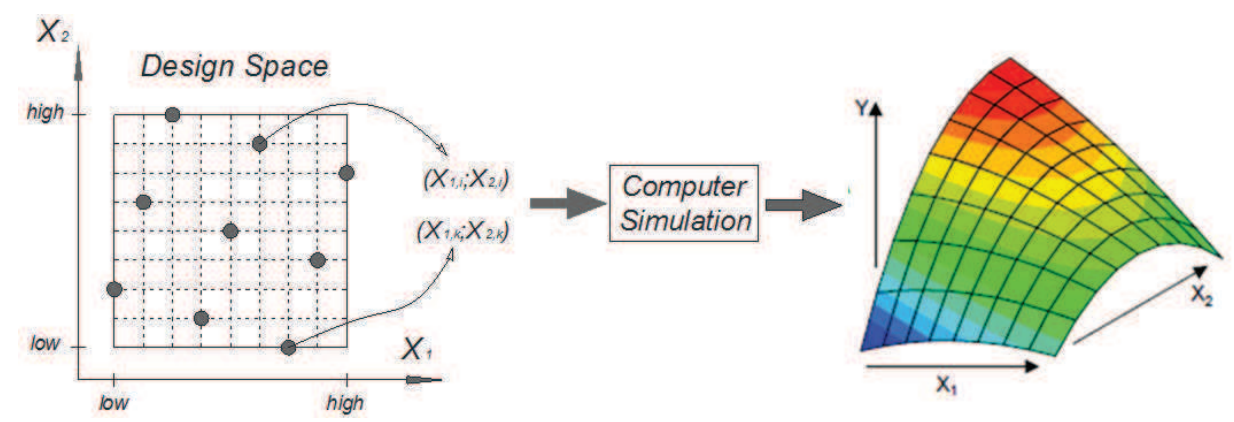

Figure 2: Principle of the computer experiment process and response surface.

This process is not necessarily linear and could be applied iteratively if the predicted model does not meet the desired accuracy. In the above process, the selection of the input variables (step 3) and the technique for approximating the response (step 4) are the two main issues that differ between physical and numerical experiments due to the deterministic property of computer experiments. These two issues will be developed in the following paragraphs.

\subsection{Selecting sampling points}

A good experimental design should minimize the number of runs needed to acquire information with a given level of accuracy. The experimental design techniques were initially developed for physical experiments. Due to the discrepancy associated with physical experimentation, classical DOEs will focus on parameter settings near the perimeter of the region of interest and take multiple data points (replicates) as shown in Fig. 3(Left). Computer experiments are determinists and are not subjected to this necessity. The objective of computer experiments is hence mainly to uniformly distribute the sampling points in the region of interest (such a design is called "space filling") as seen in Fig. 3(Right). 
Within the available methods of sampling [21], the following three are the most common and efficient: the Monte Carlo method (MC), the Latin Hypercube Sampling method (LHS) and the Quasi-Monte Carlo methods (QMC) which can be viewed as deterministic versions of MC methods because they use deterministic points rather than random samples. Blatman et al. [22] showed that QMC overperforms MC and LHS, when used with polynomial response surfaces with a mean computational gain factor of 10 in order to reach a given accuracy. The QMC methods are also termed as low discrepancy procedures: sampling points are selected in such a way that the error bound is as small as possible.
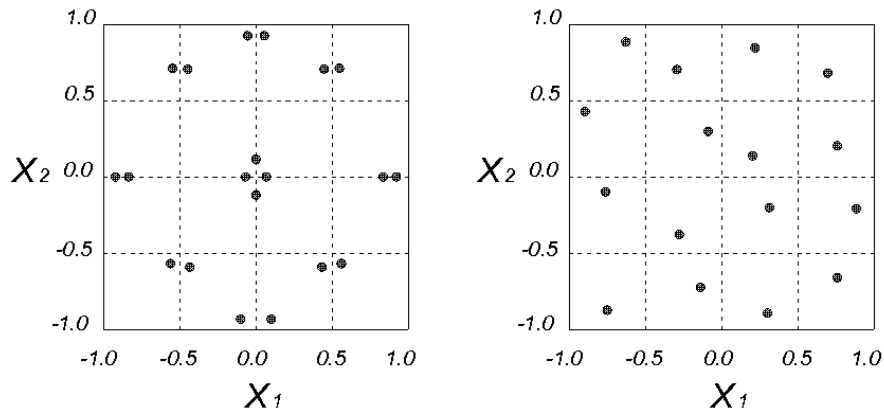

Figure 3: (Left)"Classical" and (Right) "Space Filling" designs.

There are many ways to construct a QMC sequence but the Sobol' sequences are the most widely used because they are quick to construct and fast to converge [23]. They also have the advantage of preserving the uniformity of the distribution when the dimension increases: a Sobol' sequence can be constructed from a shorter one by adding points to the shorter sequence, on the contrary to LHS, where the entire sampling process must be run again. 


\subsection{Response model regression}

After selecting the appropriate experimental points and performing the necessary computer runs, the next step is to choose an approximated model and a fitting method. The approximated model must be simple and represent adequately the response of the studied problem. In recent years, a lot of work has been done on approximated models: polynomial response surfaces, neural networks, kriging or multivariate adaptive regression splines [24]. Despite the variety of approximations that is available, comparative studies of these approaches are limited [25]. Depending on the complexity of the problem, one of the aforementioned method might be suitable. However, the polynomial response surface model is by far the simplest; it has been used efficiently in a wide variety of applications and has provided good approximated solutions to even very complex problems [26]. Beside, the use of polynomial response surface for furthers studies such as reliability [27] and optimisation [28] is relatively easy.

\subsection{Statistic tools for adequacy checking}

As mentioned in the section 2, model adequacy checking is an important part of the data analysis procedure. Indeed it is necessary to ensure that the fitted model provides an adequate approximation of the true system and to verify that none of the model assumptions is violated. In most cases, the regression model is a linear function of some unknown coefficients which are identified thanks to the least square method which will be used here for its simplicity and reliability. 


\section{Application to cylindrical curved panels under uniform axial compression}

In a former study, the authors [12] investigated the case of unstiffened cylindrical curved panels under axial compression (see Fig. 4) and proposed a set of formulas for the evaluation of the ultimate strength. These formulas were established following the general European Standards procedure for all kind of stability verification and will be used as a reference case to validate the accuracy and relevance of the methodology proposed in previous section. The strength of the panel $\chi$ was hence given as a function of the relative slenderness $\bar{\lambda}$ and three parameters $\bar{\lambda}_{0}, \beta, \alpha_{Z}$ depending on the relative curvature:

$$
\chi=\frac{2 \beta}{\beta+\bar{\lambda}+\sqrt{(\beta+\bar{\lambda})^{2}-4 \beta\left(\bar{\lambda}-\alpha_{Z}\left(\bar{\lambda}-\bar{\lambda}_{0}\right)\right)}}
$$

These simulations, as well as those which will be conducted here, involved non-linear material and second-order analyses with imperfection (GMNIA). They were conducted with the software Ansys version 13 and the standard quadrilateral 4-nodes element [29]. Panels were made of elasto-plastic steel with linear hardening as indicated in EN 1993-1.5 C.6.c) (S355, $E=210 G P a$, $\nu=0.3$ and a slope of $E / 100)$. The cylindrical panels were assumed simply supported on all edges and loaded by a uniform longitudinal compression along the curved edges. An initial imperfection with the shape of the first buckling mode and with a maximum amplitude of $1 / 200^{\text {th }}$ of the smallest edge was also added. The study was limited to square panels, so that only the thickness, the width and curvature of the panels were varied. 


\subsection{Step 1: Statement of the problem}

The aim of this step is to identify in an exhaustive manner the parameters of the problem and to select among them the ones which will have an influence on the response and which are liable of variations in practical applications. Here the quantity of interest in the panel response (the output) is the ultimate strength of the panel. Basic structural engineering tells us that it is influenced by the geometry of the panels (including their imperfections), their material properties, their boundary conditions and the nature of the loading. All these parameters could be included in the experimental program, but in this first example, the objective is to validate the method and to illustrate its pertinence, so that the same restrictions as in [12] will be observed:

- the imperfections are chosen following EN 1993-1-5 [2] (i.e. their shape is that of the first buckling mode and their amplitude is $1 / 200 t h$ of the width of the panel)

- the steel grade is S355 as generally used in modern bridges;

- the panels are simply supported on all edges;

- the longitudinal compression is uniform along the curved edges.

The only varying input factors are thus the dimensions of the panels: their length $a$, width $b$, thickness $t_{p}$ and radius of curvature $R$ (see Fig. 4). Applying the Buckingham-Vaschy's theorem, it can be demonstrated that the ratio of the ultimate strength of the panel and the yield stress $\left(\sigma_{u l t} / f_{y}\right)$ depends on three independent dimensionless parameters:

$$
\frac{\sigma_{u l t}}{f_{y}}=f\left(\frac{a}{b} ; \frac{t_{p}}{b} ; \frac{b}{R}\right)
$$




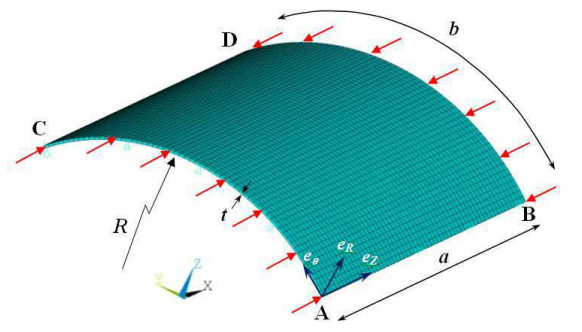

Boundary conditions:

Simple supported for 4 edges.

Unload edges $(A B \& C D)$ : free to wave

Load edges ( $\mathrm{AC} \& \mathrm{BD})$ : remain straight.

Load condition:

Uniform load compression on $\mathrm{AC} \& \mathrm{BD}$

Figure 4: Cylindrical curved panel under uniform axial compression (after [12]).

Now that the parameters have been defined, it is essential to define the range in which these parameters will vary. Every feasible configuration has to be included but the range of variation has to be kept as small as possible: it is directly linked with the precision of the approximated expression found at the end of the process. Here the ranges given in table 1 seem reasonable to cover most applications of such panels in bridge engineering.

Table 1: Design variables for unstiffened curved panels

\begin{tabular}{|c|c|c|c|}
\hline Variable & Description & Variation range & Design variable \\
\hline$a / b$ & Aspect ratio & $0.6 \leq a / b \leq 1.6$ & $X_{1}=2 a / b-2.2$ \\
$t_{p} / b$ & Slenderness & $0.01 \leq t_{p} / b \leq 0.04$ & $X_{2}=66.7 t_{p} / b-1.67$ \\
$b / R$ & Curvature (Angle) & $0 \leq b / R \leq 1$ & $X_{3}=2 b / R-1$ \\
\hline
\end{tabular}

As the order of magnitude of the variations of these three parameters is different, it is preferable to transform the physical parameters into centred variables $X_{i}$, ranging from -1 (low value) to 1 (high value). Their comparative influence on the response will hence be easier to catch. The three adimensional parameters $X_{1}, X_{2}$ and $X_{3}$ used in the coming paragraphs are thus given in the last column of table 1 . 


\subsection{Step 2: Choice of the response surface}

The choice of the response surface is based on two issues: the knowledge of the physics of the problem and the desired accuracy of the approximation. Here the target response is the ultimate strength of the plate, namely the maximum load that the plate can bear when accounting for the elasto-plastic behaviour of the material. From existing standards (EC3), it is known that the strength of a flat plate is related to the slenderness by a second order polynomial which was first proposed by Winter [30]:

$$
\frac{\sigma_{u l t}}{f_{y}}=\frac{1}{\bar{\lambda}}-\frac{0.22}{\bar{\lambda}^{2}}
$$

In (3), the slenderness $\bar{\lambda}$ is directly related to $a / b$ and $t / b$ (which means to $X_{1}$ and $X_{2}$ ) by:

$$
\bar{\lambda}=\sqrt{f_{y} \frac{12\left(1-\nu^{2}\right)}{\pi^{2} E}} \cdot \sqrt{\frac{1}{k_{a / b}}} \cdot \frac{b}{t_{p}}
$$

where $k_{a / b}$ is a function of $a / b$

$$
k_{a / b}= \begin{cases}\left(\frac{a}{b}+\frac{b}{a}\right)^{2} & \text { if } \frac{a}{b} \leq 1 \\ 4 & \text { if } \frac{a}{b} \geq 1\end{cases}
$$

It can thus be concluded that a second order polynomial should provide a good approximation of the strength of a curved plate and that it will be meaningful from a physical point of view. The response surface will thus be investigated in the following form:

$$
\widehat{Y}\left(=\frac{\sigma_{u l t}}{f_{y}}\right)=\beta_{0}+\sum_{i=1}^{3} \beta_{i} X_{i}+\sum_{j=1}^{3} \sum_{i=1}^{j} \beta_{i j} X_{i} X_{j}
$$

where $\widehat{Y}$ is the approximated response, $X_{i}$ are the three input variables and $\beta_{(.)}$are the ten unknown parameters. 


\subsection{Step 3: Selection of the input data or sampling points}

The selection of the input data points covers the choice of the number of points and of their distribution in the investigated domain which is here a hypercube in the three dimensional space. The generation of a set of sampling points using a QMC method in this cube can be made easily using a common statistical tool providing a function generating a Sobol' sequence (Matlab here). Noting that with the Sobol' sequence, the extreme values of the parameters can only be reached for an infinite number of variables, some additional points located at the corners of the cube can be added to the sequence to give more weight to the boundary of the domain.

The key issue is thus the definition of the minimal number of experiments to be conducted to get a response with the desired accuracy. The number of simulations $n$ depends on the complexity of the studied phenomenon as well as of the complexity of the approximated model. Yet there is not a unanimously agreed method relating the number of observations versus the number of independent variables in the model. Some authors suggest $3 m+1$ points [31] for a second-order polynomial approximation where $m=\frac{(p+1)(p+2)}{2}$ is the number of unknown coefficients and $p$ is the number of input variables. Following this suggestion for the present example which has 3 input parameters, a second order polynomial approximation will have 10 unknown parameters which could be evaluated with a good accuracy with 31 experiments. Adding the corner points (in total 7 additional points as the point $(-1 ;-1 ;-1)$ is by construction always part of the sequence) of the investigated domain, the total number of sampling points is set to 38 . An illustration of such a set is shown in Fig. 5. 


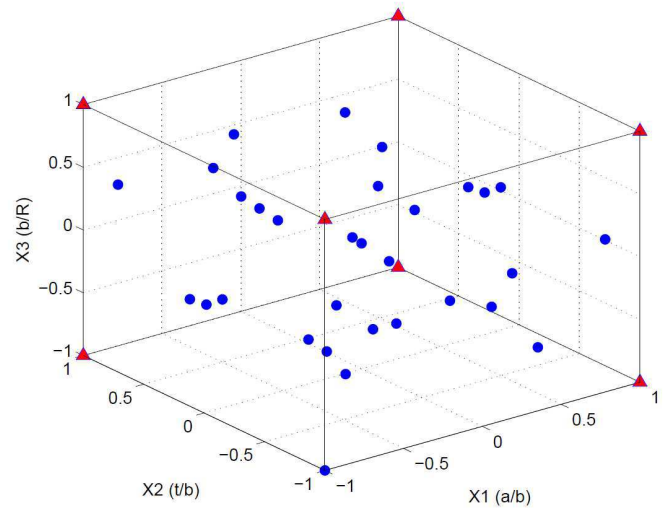

Figure 5: Sampling points generated by the Sobol' sequence (circles) and additional corner points (triangles).

\subsection{Step 4: Parameters evaluation}

The approximated response surface is here looked for in the form of a second-order polynomial in the three variables $X_{i}$ given by Eq. 6 . The unknown parameters $\beta_{(.)}$have to be identified from the numerical experiments (here $n=38$ ) which is here done by the least square method. So, from the 38 sampling points shown in Fig. 5, the ultimate strength of a curved plate can be approximated by the following expression:

$$
\begin{aligned}
\widehat{Y}= & 0.879+0.002 X_{1}+0.212 X_{2}+0.052 X_{3}-0.001 X_{1} X_{2}-0.063 X_{2} X_{3} \\
& -0.004 X_{3} X_{1}-0.037 X_{1}^{2}-0.100 X_{2}^{2}-0.003 X_{3}^{2}
\end{aligned}
$$

\subsection{Step 5: Evaluation of the accuracy of approximated model}

To evaluate the accuracy of the approximated model, conducting an analysis of variance (ANOVA) is very useful. The coefficient of determination is first determined $R^{2}=0.977$ and then the cross-validation coefficient $Q^{2}=0.969$. The fact that $R^{2}$ is very close to 1 indicates that the regression 
model fits well the data. The small difference between $R^{2}$ and $Q^{2}$ indicates that most observations have an influence on the regression equation and that the approximation model predicts well the observations. Moreover, the same model has been identified on a sample without additional points in the corners, leading to $R^{2}=0.971$ and $Q^{2}=0.942$, the small diminution of these coefficients is a direct consequence of the diminution of the number of sampling points (31 instead of 38), not to the fact that the discarded points were located in the corners of the domain. It is thus concluded that, the addition of corner points is not necessary to get an accurate estimation of the regression coefficients.

\subsection{Step 6: Selection of most significant terms and conclusion.}

The second-order formula presented in Eq. 7 for the evaluation of the ultimate strength of curved steel panels under axial compression provides a good and best possible approximation of the real capacity of the panel. However, it is remarked that not every coefficient in Eq. 7 have the same order of magnitude. So, rather than trying to explain the model with all its terms, it can naturally be asked if some terms could be excluded from the initial model without altering significantly the accuracy of the whole model.

A criterion of exclusion should hence be fixed. If normality assumptions are verified (as in the present case), the t-test provides a fully reliable criterion as it relates the value of each coefficient to its estimated standard error. More simple criteria, such as arbitrary thresholds of significance are also very effective. Indeed, as the parameters all vary between -1 and 1 , the contributions of the various terms can directly be analysed by comparing the coefficients which might then be neglected if their value is bellow a certain 
absolute value (for example $2 \%$ of the sum of the coefficients absolute values or $5 \%$ of the maximum value of the coefficients). Fixing here this threshold to $2 \%$ of the sum of the coefficients, the terms $\left(\beta_{1}, \beta_{12}, \beta_{13}\right.$ and $\left.\beta_{33}\right)$ are found not significant (a criterion based on t-test and a $90 \%$ two sided interval would give the same results). The new model is thus given by Eq. 8; it preserves good precision with high value of $R^{2}=0.976$ and $Q^{2}=0.9674$.

$$
\widehat{Y}=0.879+0.212 X_{2}+0.052 X_{3}-0.063 X_{2} X_{3}-0.037 X_{1}^{2}-0.100 X_{2}^{2}
$$

Introducing the physical parameters of table 1 into Eq. 8, the ultimate strength of unstiffened cylindrical curved panels under axial compression is given by:

$$
\begin{gathered}
\frac{\sigma_{u l t}}{f_{y}}=\left(-0.09+0.326(a / b)-0.148(a / b)^{2}\right)+(40.6+0.314 Z)\left(t_{p} / b\right) \\
-(444+8.40 Z)\left(t_{p} / b\right)^{2}
\end{gathered}
$$

where $Z$ is the curvature parameter defined by $Z=b^{2} / R t_{p}$. Eq. 9 is very similar to the classical expression of the stability problem, where the ultimate strength is represented as a polynomial function of the slenderness $t / b$.

Fig. 6 shows how well the expressions given by the DOE method (red squares) and by the semi-analytical method [12] (green triangles) are able to predict the numerical results (given by F.E. model). For most input values, the two models have less than $5 \%$ of discrepancy (in absolute value) to the true numerical value. However a few observations (No. 19, No. 22 and No. 28) predicted by DOE method have higher discrepancy (from $8 \%$ to $10 \%)$ on the contrary to the semi-analytical method whose error remain below $5.5 \%$. This might be explained by the fact that, although the semianalytical model is not richer (the calibration of $\overline{\lambda_{0}}, \beta, \alpha_{Z}$ involves only 7 


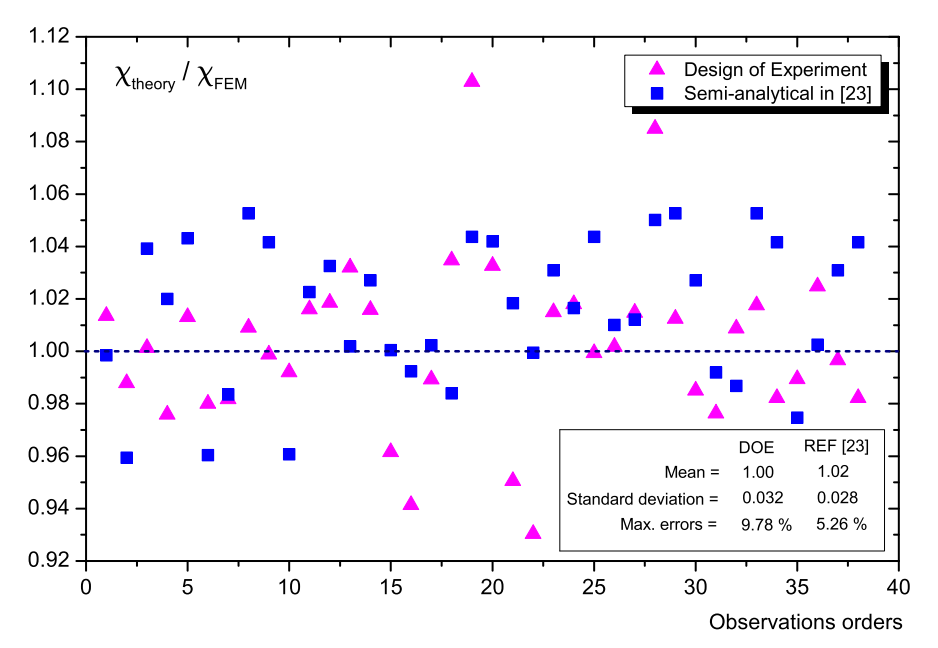

Figure 6: Comparison of FEM results with DoE and semi-analytical method [12].

parameters), its physical bases are finer which proves the crucial importance of the choice of the parameters and response surface.

(3)

These values of error should be relativised by the fact that in many complex structures such as stiffened plates, the difference between Eurocode predictions and the results of numerical simulations might reach until $20 \%$, sometimes in favor of safety, sometimes not $[32,33]$. Moreover, as the socalled characteristic value of a member is obtained by dividing its design value (e.g. Eq. 9) by some safety factor (often taken as $\gamma_{M 1}=1.1$ ) the present discrepancy is indeed acceptable. 


\section{Application to stiffened curved panels under uniform axial com- pression}

The behaviour of stiffened curved panels is a more complex problem, especially due to the interaction of different parameters (curvature, relative rigidity of stiffeners and plate, imperfection, etc.) for which no semi-analytic expression exists. So, as it has just been shown that the design of computer experiment method is well adapted for studying the stability of curved plates, it will be used for the development of a preliminary design formula (i.e suited for hand-calculation) for the ultimate resistance of stiffened curved panels.

\subsection{Finite element modelling}

The stiffened panels are modelled and analysed using the commercial finite element software Ansys [29]. The panels are supposed to be simply supported on all edges of the panel $\left(u_{r}=0\right.$ in the cylinder coordinate system of Fig. 7) but not on the stiffeners (unfavourable condition).

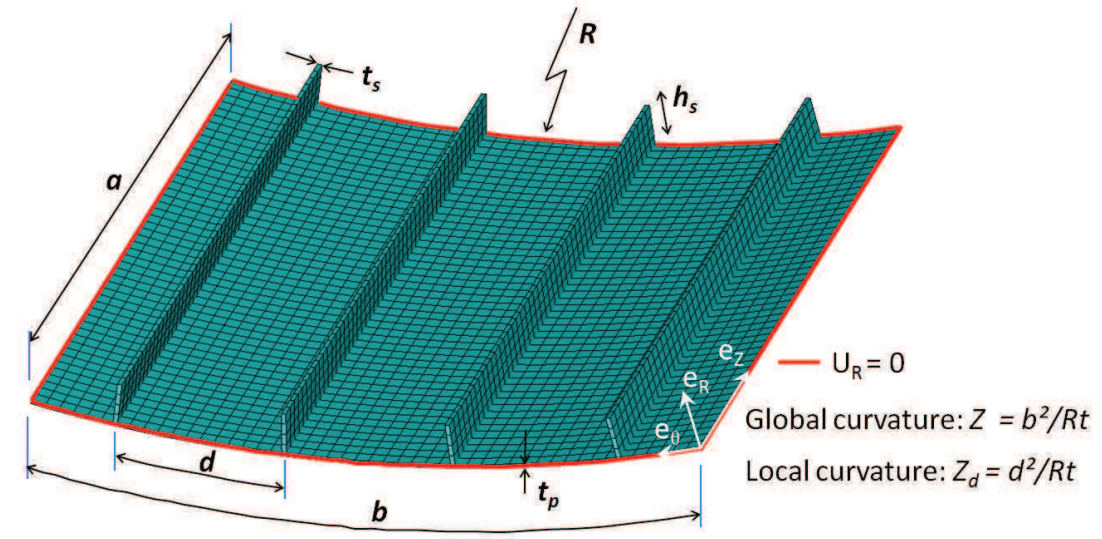

Figure 7: Boundary conditions of simply supported on all edges 
For loading conditions, the study is here limited to a uniform compression in the longitudinal direction as it is the dominant loading in bottom flange panels. It is applied not only to the main panel, but also to the stiffeners due to their participation in the overall behaviour of the structure (Fig. 8). In fact, in a bridge, the compressive forces acting on the flange come through the diaphragms and webs that connect the upper and lower panels of the box girder. By construction, the stiffeners, in most cases, are continuous and attached by welding to diaphragms: therefore they are also subjected to the compressive load.
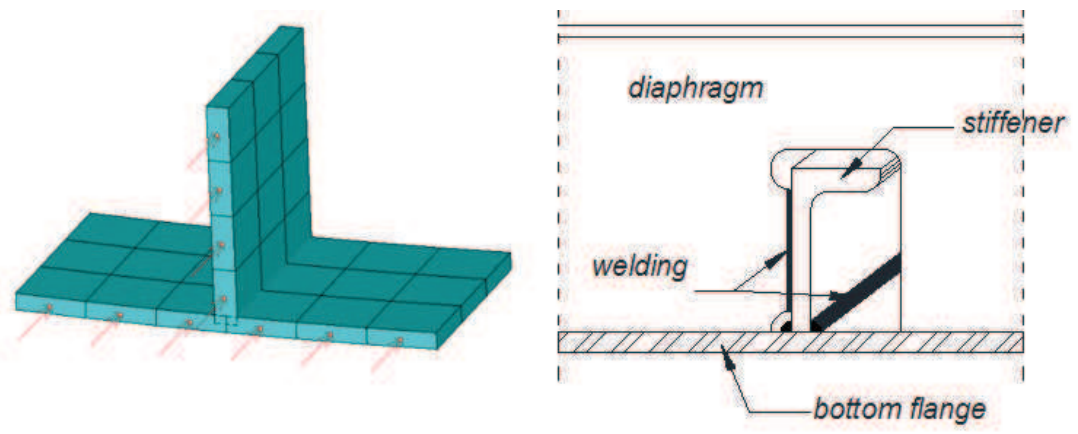

Figure 8: Loading condition and scheme of the connection stiffener/diagram by welding

The curved panels are meshed with eight-nodes shell elements which use an advanced shell formulation that accurately incorporates initial curvature effects (this element is called SHELL-281 in [29]). They are well-suited for linear, large rotation and large strain non-linear applications and offers improved accuracy in curved shell structure simulations and faster convergence than plate elements as one can see in figure 9 which represents the convergence study from [34]. A fine mesh with more than 30 elements per panel edges is used to reduce the discretisation error. 

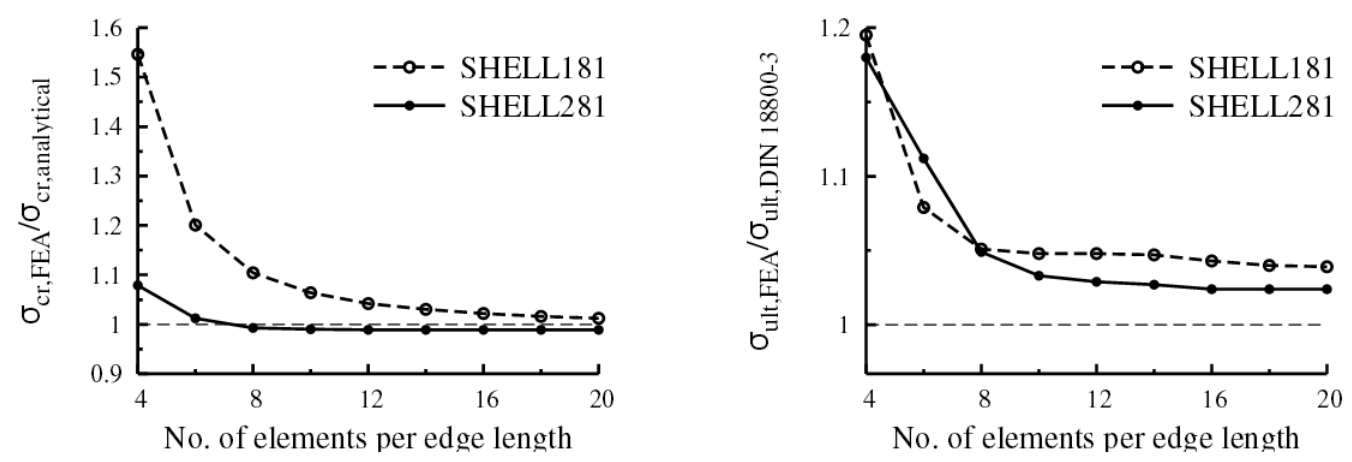

Figure 9: Convergence study of SHELL-281 element: linear bifurcation analysis (left), non linear buckling analysis (right)

The panels are all made of steel which is assumed to be elasto-plastic with linear strain hardening as indicated in EN 1993-1-5 C.6 for the material non-linear second-order analyses with initial imperfections (GMNIA). The Young modulus E and Poisson's ratio are taken equal to $210 \mathrm{GPa}$ and 0.3 respectively. The steel grade is $\mathrm{S} 355$ with a yield steels equal to $355 \mathrm{MPa}$.

\subsection{Evaluation of the ultimate strength}

This study is limited to the case of stiffened curved panels under axial compression with open section stiffeners (simple flat plates) because the curvature makes it difficult to realise a close form section of stiffener (boxed rib). Therefore, the number of input parameters is here restricted to seven as presented in table 2 . The ranges of variation of these parameters are chosen in order to cover most panels used in bridge construction. As the orders of magnitude of the parameters variations are different, they are transformed the physical parameters into centred variables $X_{i}$, ranging from -1 to 1 . 
Table 2: Design variable (dimension in meter)

\begin{tabular}{|c|l|c|l|}
\hline Variable & Description & Variation range & Design variable \\
\hline$a$ & Length of the panel & $4 \leq a \leq 6$ & $X_{1}=a-5$ \\
$b$ & Width of the panel & $4 \leq b \leq 6$ & $X_{2}=b-5$ \\
$t_{p}$ & Thickness of panel & $0.01 \leq t_{p} \leq 0.02$ & $X_{3}=200 \cdot t_{p}-3$ \\
$1 / R$ & Curvature of panel & $0 \leq 1 / R \leq 0.1$ & $X_{4}=20 / R-1$ \\
$d$ & Distance between stiffeners & $0.3 \leq d \leq 0.8$ & $X_{5}=4 \cdot d-2.2$ \\
$h_{s}$ & Height of stiffener & $0.1 \leq h_{s} \leq 0.2$ & $X_{6}=20 \cdot h_{s}-3$ \\
$t_{s}$ & Thickness of stiffener & $0.01 \leq t_{s} \leq 0.02$ & $X_{7}=200 \cdot t_{s}-3$ \\
\hline
\end{tabular}

$$
\begin{aligned}
& -0.47 X_{1}+3.58 X_{2}+4.24 X_{3}+7.32 X_{4}-3.87 X_{5}+4.83 X_{6}+2.33 X_{7} \\
& +1.65 X_{2} X_{4}-1.72 X_{2} X_{5}+1.71 X_{2} X_{6}+0.89 X_{2} X_{7}+1.33 X_{3} X_{4} \\
& -0.76 X_{3} X_{5}-1.73 X_{4} X_{5}+0.81 X_{4} X_{6}-1.18 X_{5} X_{6}+0.94 X_{6} X_{7}
\end{aligned}
$$


The coefficient of determination $R^{2}=0.986$ (close to 1 ) indicates that the value predicted by the model fits very well the data (see Fig. 10). Also, the small difference between the values of $Q^{2}=0.978$ and $R^{2}$ shows that there are few undue influence on the regression equation. The accuracy of the response is also checked by the relative mean absolute error (RMAE): $4.2 \%$ (which indicated that a mean error of $4 \%$ is expected), the relative minimum and maximum error: $-14.0 \%$ and $+13.5 \%$ respectively. The above equation is thus fairly acceptable in bridge constructions and residual errors can be easily covered by using a safety factor.

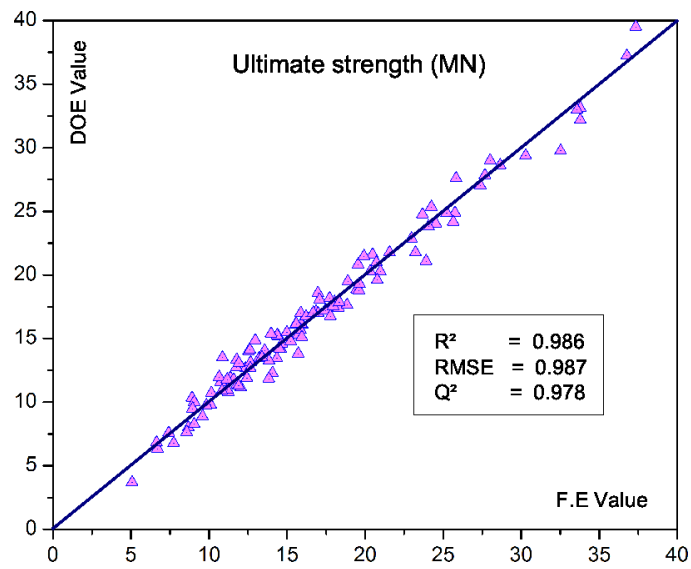

Figure 10: Comparison of the ultimate strength of the FEM and DOE

Considering now every coefficients independently, it is found that the $5 \%$ two-sided confidence interval of the regression coefficients is $\pm 0.20 \mathrm{MN}$ for the constant term, $\pm 0.36 M N$ for the linear terms and $\pm 0.62 M N$ for the quadratic terms. As generally observed, the uncertainty on linear terms is almost twice smaller than that on quadratic terms: the direct influences of the parameters are better known than those of their interactions. 
These statistical remarks being made, it is remarkable that all parameters are found significant in Eq. 10. In decreasing order, the most significant parameters are the curvature $\left(X_{4}\right)$, the height of the stiffeners $\left(X_{6}\right)$, the thickness of the panel $\left(X_{3}\right)$, the distance between stiffeners $\left(X_{5}\right)$, the width of the panel $\left(X_{2}\right)$, the thickness of the stiffeners $\left(X_{7}\right)$ and finally the length of the panel $\left(X_{1}\right)$ whose influence is very limited (no more than $\pm 3 \%$ of the total strength). Quite obviously, increasing the curvature, the thickness of the plate or the height and thickness of the stiffeners increases the strength of the panel, while increasing the length of the panel or the distance between stiffeners decreases it. Then the fact that the strength grows with the width is not so immediate but can be easily understood considering that when the width of the panel increases, the distance between the centre of gravity of the panel and the curved plate increases due to curvature and by there the global inertia of the curved panel increases.

There are then multiple interactions which combine effects are more difficult to analyse. Indeed increasing the curvature, the height and thickness of the stiffeners or the thickness of the panel has always a positive effect on the strength because, for these parameters, the linear term dominates clearly the quadratic terms. Then concerning the distance between the stiffeners, in most cases diminishing it leads to an increase of the strength but not mandatory as for slender panels with small curvature and small stiffeners it might lead to a smaller strength (indeed the coefficient of $X_{5}$ is $-3.87-1.72 X_{2}-0.76 X_{3}-1.73 X_{4}-1.18 X_{6}$ and varies between $-9.26 M N$ and $1.52 \mathrm{MN})$. In the same way, in most cases increasing the width of the panel leads to an increased strength but not for slender panels when the spac- 
ing between stiffeners is too large (the coefficient of $X_{2}$ being $3.58+1.65 X_{4}-$ $1.72 X_{5}+1.71 X_{6}+0.89 X_{7}$, it varies between $-2.39 M N$ and $\left.9.55 M N\right)$. Very likely in the last two cases, these changes of the coefficient sign correspond to a change in the buckling mode from column to plate or from global to local.

Anyway, it must be recalled that even in these extreme cases, the error in the prediction of the strength is not larger than in other cases (cf. Fig. 10) and that according to all statistical criteria mentioned above, the approximated model given by expression (10)) is able to predict correctly the ultimate strength of stiffened curved panels in the interested domain. It can thus be easily inserted in an optimisation pattern as illustrated in the coming section.

\subsection{Cost optimisation of curved stiffened panels}

The cost optimisation scheme proposed here is based on a cost objective function similar to the ones used by [35]. It assumes that the manufacturing cost of a stiffened curved panel $K$ defined by the parameters $X_{i}$ is the sum of the material costs $K_{m}$ (the steel cost) and of the fabrication costs $K_{f}$ which can be defined as follow:

$$
K\left(X_{i}\right)=K_{m}+K_{f}=k_{m} \rho V+k_{f} \sum T_{i}
$$

where $\rho$ is the steel density, $V$ is the total volume of the curved panel, $k_{m}$ and $k_{f}$ are characteristic coefficients of material and fabrication costs. $T_{i}$ denotes manufacturing times:

- $T_{1}$ : time for preparing, cutting and assembling the pieces:

$$
T_{1}=\Theta_{d} \sqrt{\kappa \rho V}
$$

with $\Theta_{d}$ a factor characterising the impediment for welding and $\kappa$ the number of elementary pieces to be welded; 
- $T_{2}$ : time for welding and $T_{3}$ : additional time for maintenance of the machine which might be considered as $0.3 T_{2}$, so that:

$$
T_{2}+T_{3}=1.3 \sum C_{i} a_{w i}^{2} L_{w i}
$$

where $L_{w i}$ is the length of the $\mathrm{i}^{\text {th }}$ weld, $a_{w i}=\max \left(0.4 t_{s}, 4 m m\right)$ its width and $C_{i}$ a coefficient depending on the welding technique which is here taken equal to 0.2349 for Shielded Metal Arc Welding.

The constraint equation is then given by the stability requirement of the panel:

$$
g\left(X_{i}\right)=\frac{N_{a p p}}{N_{u l t} / \gamma_{M 1}}-1 \leqslant 0
$$

where $N_{a p p}$ is the applied load, $N_{u l t}$ the capacity of the panel estimated by Eq. (10) and $\gamma_{M 1}$ is a safety factor.

The panel which is proposed here for optimisation has fixed overall dimensions: its length $a$ is $6 \mathrm{~m}$, its width $b$ is $4 \mathrm{~m}$ and its curvature radius $R$ is $20 \mathrm{~m}$ ). It is subjected to a uniform axial compression $N_{a p p}=12 \mathrm{MN}$. The objective is thus to determine the parameters (thickness of the panel $t_{p}$, thickness $t_{s}$ and height $h_{s}$ of the stiffeners and distance between stiffeners $d$ ) which will minimize the cost of the panel (11) and verify the constraint equation (14). To make the problem more realistic, it is also considered that the variables are not continuous but discrete (which poses no problem to Matlab optimisation algorithm), so that the solution is looked for in the following domain:

- $t_{p} \in[0.01 ; 0.02]$ by steps of $1 \mathrm{~mm}$;

- $d \in[0.3 ; 0.8]$ by steps of $5 \mathrm{~cm}$; 
- $h_{s} \in[0.1 ; 0.2]$ by steps of $1 \mathrm{~cm}$;

- $t_{s} \in[0.01 ; 0.02]$ by steps of $1 \mathrm{~mm}$;

Concerning then the definition of cost coefficients, as no precise data were available for $k_{f}$ and $k_{m}$, it was decided to present the results in an adimensional form considering different values of the ratio $k_{f} / k_{m}$. For $k_{f} / k_{m}=0$, only material cost is taken into account, while for large values of $k_{f} / k_{m}$, manufacturing cost prevail (reasonable values in northern countries lie between 1 and 2). The results of the optimisation procedure are shown in table 4.3 (where $n$ is the total number of stiffeners).

Table 3: Results of the optimisation procedure for $N_{a p p}=12 M N$

\begin{tabular}{|c|c|c|c|c|c|c|}
\hline$k_{f} / k_{m}$ & $t_{p}$ & $d$ & $n$ & $h_{s}$ & $t_{s}$ & $K$ \\
\hline 0.0 & 0.014 & 0.55 & 7 & 0.16 & 0.015 & 3450 \\
0.5 & 0.015 & 0.75 & 5 & 0.18 & 0.016 & 4250 \\
1.0 & 0.016 & 0.90 & 4 & 0.19 & 0.017 & 5000 \\
2.0 & 0.018 & 0.90 & 4 & 0.17 & 0.015 & 6200 \\
\hline
\end{tabular}

About the method first, it must be noticed here that the set of optimised parameters corresponding to each ratio $k_{f} / k_{m}$ was obtained almost immediately thanks to the preliminary design formula developed in section 4.2 whereas it would have taken hours or even days using directly finite element simulations. Concerning the results then, following remarks can be drawn:

- The number of stiffeners is higher when only material costs are considered. It is however not maximal $\left(n_{\max }=12\right)$ which shows that 
increasing reasonably the thickness of the plate is very efficient from a weight point of view.

- For higher values of $k_{f} / k_{m}$, stiffeners becomes logically stiffer to reduce their number and the number of welds.

- It is often more economical to increase the panel thickness than to increase the number of stiffeners which confirms the conclusion of [33].

\section{Conclusion}

Stiffened curved panels in civil engineering structures have high sensitivity to instability phenomenon. Analytical or semi-analytical studies are often not feasible as the problem depends on many parameters such as the panel's curvature or the panel configuration with its stiffener and semi-rigid supports. There is hence a need for a robust strategy when attempting to develop approximated models for such problems. The proposition of such a strategy was the aim of the first part of the present paper and this, through a turnkey methodology based on the theory of the design of experiment method. The efficiency of the method was first reviewed. Some particular points which differentiate the ordinary physical experiments from computer experiments were discussed. Afterwards this methodology was applied to the case of unstiffened curved panels for which solutions were already available in order to evaluate the accuracy of the method and its relevance. A huge gain of time was noticed when using the DOE method: only 38 simulations were needed in the first application against 524 observations in [12] for determining the capacity of a curved panel. Also the general accuracy of the model in the 
form of a second-order polynomial was comparable to that obtained with more standard heuristic methods. Moreover, as the experiment designer had existing knowledge of the problem, the input values were adequately chosen and the physical interpretation of the results was easy and satisfying, despite the simplicity of the model. The strategy proposed here provides thus a reliable alternative method for the prediction of the ultimate strength of curved panels.

Confident in the methodology, the authors then developed a fully reliable formula for preliminary design of stiffened curved panels. The accuracy of the formula was demonstrated and the influence of various design parameters was discussed. A simple cost optimisation problem was finally presented to illustrate the potential of the formula.

\section{Acknowledgement}

The authors would like to acknowledge here Prof. B. Sudret from ETH Zürich for the kind advices that he provided during the maturation of this paper. 


\section{Nomenclature}

${ }_{528} \quad \alpha_{Z} \quad$ Parameter characterising the imperfections sensitivity

${ }_{529} \quad \beta \quad$ Parameter characterising the asymptotic behaviour of the panel

${ }_{530} \quad \beta_{0} \quad$ Constant term and average value of the approximated response

${ }_{531} \beta_{i} \quad$ Coefficient characterising the effect of the variable $X_{i}$

${ }^{532} \beta_{i j} \quad$ Coefficient characterising the interaction of the variables $X_{i}$ and $X_{j}$

${ }_{533} \chi \quad$ Reduction factor for the panel buckling according to EC3

${ }_{534} \bar{\lambda} \quad$ Relative slenderness of the panel according to EC3

${ }_{535} \quad \bar{\lambda}_{0} \quad$ Slenderness separating plastic buckling from elasto-plastic buckling

${ }_{536} \rho \quad$ Steel density

${ }_{537} \sigma_{\text {ult }} \quad$ Ultimate strength of the panel

${ }_{538} \quad a \quad$ Length of the panel

${ }_{539} \quad b \quad$ Width of the panel

${ }_{540} d \quad$ Distance between stiffeners

${ }^{541} f_{y} \quad$ Yield stress of the panel

${ }_{542} \quad h_{s} \quad$ Height of stiffeners

${ }^{543} K_{f} \quad$ Fabrication costs

${ }_{544} \quad k_{f} \quad$ Fabrication cost per volume unit 
${ }_{545} K_{m} \quad$ Material costs (steel cost)

${ }_{546} \quad k_{m} \quad$ Material cost per volume unit

${ }_{547} k_{a / b} \quad$ Parameter characterising the influence of the aspect ratio

${ }_{548} m$ Number of unknown coefficients in the approximated model

${ }_{549} n \quad$ Number of simulations or numerical experiments

${ }_{550} \quad N_{a p p} \quad$ Normal force applied to the panel

${ }_{551} N_{\text {ult }} \quad$ Capacity of the panel

${ }_{552} p \quad$ Number of input variables

${ }_{553} \quad R \quad$ Curvature radius of the panel

${ }_{554} T_{i} \quad$ Manufaturing time of the $i^{\text {th }}$ operation

${ }_{555} t_{p} \quad$ Thickness of the panel

${ }_{556} \quad t_{s} \quad$ thickness of stiffeners

${ }_{557} \quad V \quad$ Total volume of the curved panel

${ }_{558} X_{i} \quad$ Generic name of the $i^{\text {th }}$ input variable

${ }_{559} X_{i}^{j} \quad j^{\text {th }}$ value of the $i^{\text {th }}$ input variable

${ }_{560} Y, \hat{Y}$ Response and approximated response

${ }_{561} Y^{j} \quad j^{t h}$ value of the response

${ }_{562} Z \quad$ Curvature parameter defined by $Z=b^{2} / R t_{p}$ 


\section{References}

[1] Lundquist E. Comparison of three methods for calculating the compressive strength of flat and slightly curved sheet and stiffener combinations. Tech. Rep.; Nat. Advisory Committee for Aeronautics, TC 455; 1933.

[2] EN1993-1-5 . Eurocode 3 design of steel structures part 1-5: Plated structural elements. 2007.

[3] EN1993-1-6 . Eurocode 3 design of steel structures part 1-6: Strength and stability of shell structures. 2007.

[4] Batdorf S, Schildcrout M. Critical axial-compressive stress of a curved rectangular panel with a central chordwise stiffener. Tech. Rep.; 1948.

[5] Schildcrout M, Stein M. Critical axial-compressive stress of a curved rectangular panel with a central longitudinal stiffener. Tech. Rep.; NACA Technical Note 1879; 1949.

[6] Becker H. Handbook of structural stability. Part VI: Strength of stiffened curved plates ans shells. Tech. Rep.; New York University, Washington; 1958.

[7] Gall H. Compressive strength of stiffened sheets of aluminum alloy. Ph.D. thesis; Massachusetts Institute of Technology; 1930.

[8] Ramberg W, Levy S, Fienup K. Effect of curvature on strength of axially loaded sheet-stringer panels. Tech. Rep.; NACA-Technical note $944 ; 1944$. 
[9] Cho S, Park H, Kim H, Seo J. Experimental and numerical investigations on the ultimate strength of curved stiffened plates. In: Proc. $10^{\text {th }}$ Int. Symp. Practical design of ships and oth. floating str. 2007,.

[10] Khedmati M, Edalat P. A numerical investigation into the effects of parabolic curvature on the buckling strength and behaviour of stiffened plates under in-plane compression. Latin American J of Solids and Str $2010 ; 7(3)$.

[11] Park J, Iijima K, Yao T. Estimation of buckling and collapse behaviours of stiffened curved plates under compressive load. International Society of Offshore and Polar Engineers, USA; 2008,.

[12] Tran K, Davaine L, Douthe C, Sab K. Stability of curved panels under uniform axial compression. Journal of Constructional Steel Research 2012;69(1):30-8.

[13] Martins J, da Silva LS, Reis A. Eigenvalue analysis of cylindrically curved panels under compressive stresses - extension of rules from $\{\mathrm{EN}\}$ 1993-1-5. Thin-Walled Structures 2013;68:183 -94.

[14] Fisher R. The design of experiments. Edinburgh, Scotland: Oliver and Boyd; 1935.

[15] Kleijnen J. Design and analysis of computational experiments: Overview. Experimental Methods for the Analysis of Optimization Algorithms 2010;:51-77.

[16] Taguchi G, Chowdhury S, Wu Y. Taguchi's quality engineering handbook. John Wiley and Sons Hoboken, NJ; 2005. 
[17] Sacks J, Welch W, Mitchell T, Wynn H. Design and analysis of computer experiments. Statistical science 1989;4(4):409-23.

[18] Jourdan A. Design of numerical experiments. Département de Mathématiques - Revue MODULAD 2005;(in French).

[19] Santner T, Williams B, Notz W. The design and analysis of computer experiments. Springer Verlag; 2003.

[20] Montgomery D. Design and analysis of experiments. John Wiley and Sons Inc; 2008.

[21] Franco J. Design of numerical experiments in exploratory stage for complex phenomena. Ph.D. thesis; Ecole Nationale Supérieure des Mines; 2008. (in French).

[22] Blatman G, Sudret B, Berveiller M. Quasi random numbers in stochastic finite element analysis. Mechanics and Industries 2007;8(3):289-97.

[23] Krykova I. Evaluating of path-dependent securities with low discrepancy methods. Ph.D. thesis; Worcester Polytechnic Institute; 2003.

[24] Simpson T, Poplinski J, Koch P, Allen J. Metamodels for computerbased engineering design: survey and recommendations. Engineering with computers 2001;17(2):129-50.

[25] Simpson T, Lin D, Chen W. Sampling strategies for computer experiments: design and analysis. International Journal of Reliability and Applications 2001;2(3):209-40. 
[26] Allen T, Bernshteyn M, Kabiri-Bamoradin K. Constructing metamodels for computer experiments. J Quality Technology 2003;35:264-74.

[27] Zheng Y, Das PK. Improved response surface method and its application to stiffened plate reliability analysis. Engineering Structures 2000;22(5):544-51.

[28] Roux W, Stander N, Haftka R. Response surface approximations for structural optimization. International Journal for Numerical Methods in Engineering 1998;42(3):517-34.

[29] ANSYS . User's theory manual v13. 2009.

[30] Winter G. Strength of thin steel compression flanges. Transaction ASCE 1947;112:527-54.

[31] Bowman K, Sacks J, Chang Y. Design and analysis of numerical experiments. Journal of the atmospheric sciences 1993;50(9):1267-78.

[32] André I, Degée H, De Ville De Goyet V, Maquoi R. Effect of initial imperfections in numerical simulations of collapse behaviour of stiffened plates under compression. In: Proceedings of the Third European Conference on Steel Structures. 2002, p. 503-12.

[33] De Ville De Goyet V, Maquoi R, Bachy F, André I. Ultimate load of stiffened compressed plates: Effects of some parameters and discussion concerning the ec3 rules. In: Proceedings of the Third European Conference on Steel Structures; vol. 1. 2002, p. 591-600. 
[34] Braun B. Stability of steel plates under combined loading. Ph.D. thesis; University of Stuttgart; 2010.

[35] Jarmai K, Snyman J, Farkas J. Minimum cost design of a welded orthogonally stiffened cylindrical shell. Computers \& Structures 2006;84(12):787 -97. 